\title{
Fate of sciatic nerve palsy following hip arthroplasty
}

\author{
Woong Chae $\mathrm{Na}^{1 *}$, Jeong Hoon $\mathrm{Kang}^{2 *}$, Jung Woo Lee ${ }^{3 *}$, Suenghwan $\mathrm{Jo}^{3 *}$ \\ Department of Orthopedic Surgery, 'Gwanju Suwan Hospital, Gwanju, ${ }^{2}$ Mokpo Christian Hospital, Mokpo, ${ }^{3}$ Chosun University Hospital, \\ Gwangju, Korea
}

Received October 1, 2018

Revised November 12, 2018

Accepted November 13, 2018

Corresponding author

Suenghwan Jo

Department of Orthopedic Surgery,

Chosun University Hospital, 365

Pilmundae-ro, Dong-gu, Gwangju

61453, Korea

Tel: +82-62-220-3147

Fax: +82-62-226-3379

E-mail: jo.suenghwan@chosun.ac.kr

ORCID:

http://orcid.org/0000-0001-7262-710X

*These authors contributed equally

to this work.

\begin{abstract}
Sciatic nerve palsy is a rare complication that occur after total hip arthroplasty but clinically this can result in significant functional deficit. The previous literature suggests most of the sciatic nerve palsy symptom to improve after a few weeks to a few months but in the real clinical setting, the result may vary and the symptom maybe significantly prolonged and some may not fully recover to the original state. Therefore, the current study aims assessed the fate of the patients who developed sciatic nerve palsy from hip arthroplasty operation. The authors have also looked at potential risk factors for poor outcome. This is the first case series in the Korean literature and we are reporting the result with the literature review.
\end{abstract}

Keywords: Sciatic nerve; Palsy; Hip; Arthroplasty

\section{INTRODUCTION}

Sciatic nerve palsy is a rare complication that occur after total hip arthroplasty with reported incidence of 0.09 to $3.7 \%[1,2]$. While the incidence is low, clinically this can result in significant functional deficit as the main symptom of the sciatic nerve palsy is foot drop and this will result in rehabilitation delay. The previous literature suggests most of the sciatic nerve palsy symptom to improve after a few weeks to a few months and recommend protected ambulation until symptom resolve [3]. In contrast to the previous results, in the real clinical setting, the result may vary and sciatic nerve symptom maybe significantly prolonged and some may not fully recover to the original state [4-6]. How- ever, there are only limited reports on the fate of sciatic nerve palsy following the hip arthroplasty. Therefore, in this study, we assessed the fate of the patients who developed sciatic nerve palsy from hip arthroplasty operation. We believe this is the first case series in the Korean literature, thus we are reporting the result with the literature review.

\section{MATERIALS AND METHODS}

The cohort of the current study was selected with review of the hospital records. Between January 2007 and December 2015, Twenty five patients were initially identified to have clinical symptoms of sciatic nerve palsy following primary hip arthroplasty. The clinical symptom includes 
numbness at dorsal, plantar, and lateral aspects of the foot as well as over the posterior aspect of calf and thigh, and motor weakness of ankle dorsiflexion. The final diagnosis of sciatic nerve palsy was made by performing EMG (electromyography) /NCV (nerve conduction velocity) which was done at mean of 8.4 months from the surgery (range 6.3 to 14.2 months). Of the 25 patients initially selected, 19 patients were confirmed with the diagnosis of sciatic nerve palsy and these patients constitute the bases of our study (Fig. 1). The mean age of the patients were 57.9 years (range 39 to 81 years) and included 8 males and 11 females. In all patients, the operation was done using posterolateral approach. The first symptom was noticed immediately following the operation in 15 patients while in the 4 patients, the symptom was first recognize during the first routing office visit which was made at 1 month following the surgery. In all patients, initial treatment was to apply short leg splint or cast to prevent the patient from developing joint contracture or slip down during initial walking rehabilitation. The patients were fol-

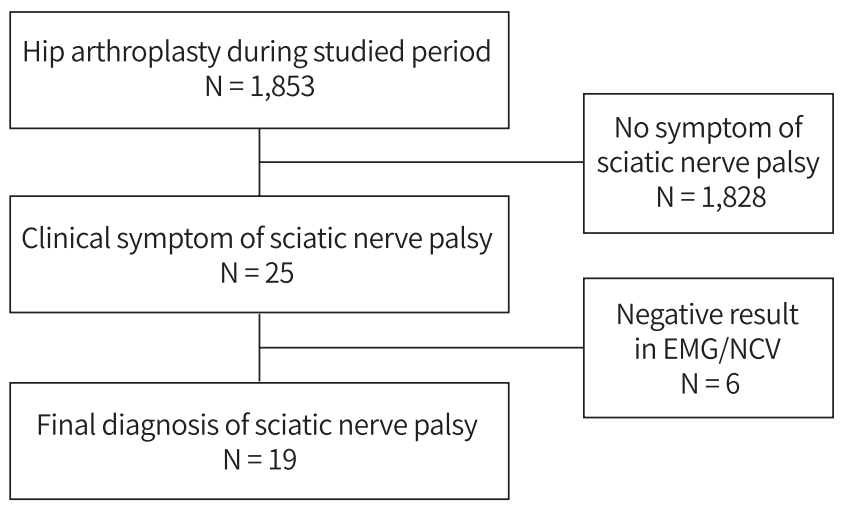

Fig. 1. Flowchart for inclusion and exclusion of studied cohort. EMG: electromyography, NCV: nerve conduction velocity. lowed at routine bases with minimum of 2 years or until the symptom resolved. The patient's motor and sensory symptoms were checked using motor grade scale (Table 1). Of the patients who did not recover, the final interview was done using telephone call or during the office visit. The status of the ambulation at the last follow up was also monitored. To assess the factors that may contribute to the improvement or worsening of the neurologic symptom, we investigate the time to operation, surgery time duration, age, gender, body mass index (BMI) and component position including offset. The potential risk factors were selected based on previous literatures and discussion among the authors. All of the listed risk factors were checked using hospital record except for the offset of which was measured using institution's PACs system (Table 2).

Statistic evaluation was performed using SPSS 18.0 software (SPSS Inc., Chicago, IL, USA). Due to the lack of sufficient number of the cohort, only the univariate analysis was performed to assess the factors that may contribute to the complete resolution of the symptom. A $p$ value of $<0.05$ was considered significant in all analysis.

Table 1. Motor grade scale

\begin{tabular}{cl}
\hline Grading & \multicolumn{1}{c}{ Description } \\
\hline 0 & Total paralysis \\
1 & Palpable or visible contraction \\
2 & Active movement, full range of motion, gravity eliminated \\
3 & Active movement, full range of motion, against gravity \\
4 & Active movement, full range of motion, against gravity and \\
& provides some resistance \\
5 & Active movement, full range of motion, against gravity and \\
& provides normal resistance \\
\hline
\end{tabular}

Table 2. Statistical analysis of relevant factors

\begin{tabular}{lccc}
\multicolumn{1}{c}{ Risk factors } & Overall & $\begin{array}{c}\text { Complete resolution group } \\
(\mathrm{N}=11)\end{array}$ & $\begin{array}{c}\text { Incomplete resolution group } \\
(\mathrm{N}=8)\end{array}$ \\
\hline Time to operation (d) & $4.5 \pm 3.2$ & $4.2 \pm 2.3$ & $4.7 \pm 2.6$ \\
Surgery time duration (min) & $49.1 \pm 22.3$ & $48.3 \pm 20.9$ & $50.2 \pm 22.3$ \\
Age (y) & $57.9 \pm 32.3$ & $55.3 \pm 29.3$ & $59.2 \pm 24.3$ \\
Gender (male:female) & $8: 11$ & $5: 6$ & $3: 5$ \\
Body mass index & $27.0 \pm 3.8$ & $27.2 \pm 3.2$ & $26.9 \pm 2.9$ \\
Cup position within safe zone & $89 \%$ & $87 \%$ & $93 \%$ \\
Horizontal offset difference $(\mathrm{mm})$ & $+3.4 \pm 2.1$ & $+3.6 \pm 1.8$ & $+3.2 \pm 1.6$ \\
Vertical offset difference $(\mathrm{mm})$ & $+4.8 \pm 3.4$ & $+4.6 \pm 3.8$ & $+5.1 \pm 2.8$ \\
\hline
\end{tabular}

Values are presented as mean \pm standard deviation or number only or percent. 


\section{RESULTS}

The mean follow-up period was 38 months which ranged from 11 to 180 months. None of the patient had any non-neurologic complications related to the total hip arthroplasty which includes instability, infection or prosthesis failure. At the initial observation, all patients had numbness at dorsum of foot region. Regarding the index motor grade, 5 patients had motor weakness of grade 0,7 patients had grade 1, 3 patients had grade 2 and 4 patients had grade 3. The complete resolution was noticed in 11 patients. In these patients, the mean time to full recovery was at 4.5 months from the index surgery. A partial improvement, as defined by increase in motor grade, was observed in 6 patients. The patient with no improvement was noticed in 2 patients which was followed upto 48.2 months. Of these 8 patients who had only the partial or no recovery, decrease in ambulatory status as compare to the preoperative state was by 1 grade in 3,2 grade in 3 and 3 grade in 2 patients. The univariate analysis showed that the young age was the only factor contributing to the improvement of the symptom (Table 3).

\section{DISCUSSION}

In contrast to the previous belief [3], the result of the current study shows that significant percentage of the sciatic nerve palsy that occur following the hip arthroplasty do not fully recover. The study demonstrates that the sciatic nerve palsy that occur in old age patient are the risk factor for negative result. This is consistent with the study by Park

Table 3. Univariate analysis associated with sciatic nerve symptom recovery

\begin{tabular}{lcc}
\hline \multicolumn{1}{c}{ Risk factors } & $\begin{array}{c}\text { Hazard ratio for recovery } \\
(95 \% \mathrm{Cl})\end{array}$ & $\mathrm{p} \mathrm{value}$ \\
\hline Time to operation & $1.38(0.70-2.13)$ & 0.428 \\
Surgery time duration & $1.00(0.41-2.05)$ & 0.235 \\
Age & $0.85(0.75-0.98)$ & $0.042^{\mathrm{a}}$ \\
Gender & $1.08(0.65-1.75)$ & 0.331 \\
Body mass index & $0.75(0.41-1.45)$ & 0.241 \\
Cup position within safe zone & $1.73(0.56-3.24)$ & 0.587 \\
Horizontal offset difference & $1.01(0.11-4.26)$ & 0.368 \\
Vertical offset difference & $1.28(0.34-3.21)$ & 0.681 \\
\hline
\end{tabular}

${ }^{a} p$ value with statistical significance. et al. [5] who also reported similar conclusions. However, unlike their report, we were unable to find the correlation between the symptom resolution and the BMI. We believe the reason why the BMI was not a factor in our series is that none of the patients in our cohort were obese while significant number of obese patients were included in the study by Park et al.

Although rarely reported, the sciatic nerve palsy is one of the complication following total hip arthroplasty that may significantly influence the patient outcome $[7,8]$. The reason for developing sciatic nerve palsy is controversial but theoretically any direct or indirect interference in the course of the sciatic nerve can be a risk factor [8]. Edwards et al. reported $1.1 \%$ of sciatic nerve palsy following the hip arthroplasty and suggested that the direct or indirect injury by the retractor maybe have caused a nerve palsy [9]. We noticed that in all our cases the sharp Homan retractor was utilized to retract the posterior musculotendionous structure where the sciatic nerve is in proximity and this may have been a reason for sciatic nerve damage in our series. Another potential factor would be postoperative hematoma. Butt et al. reported 6 cases of sciatic nerve palsy that occurred following the hip arthroplasty and suggested that evacuation of the hematoma maybe necessary in unresolved cases [2]. However, we think this may not be the problem in our series as the absence of hematoma was confirmed with

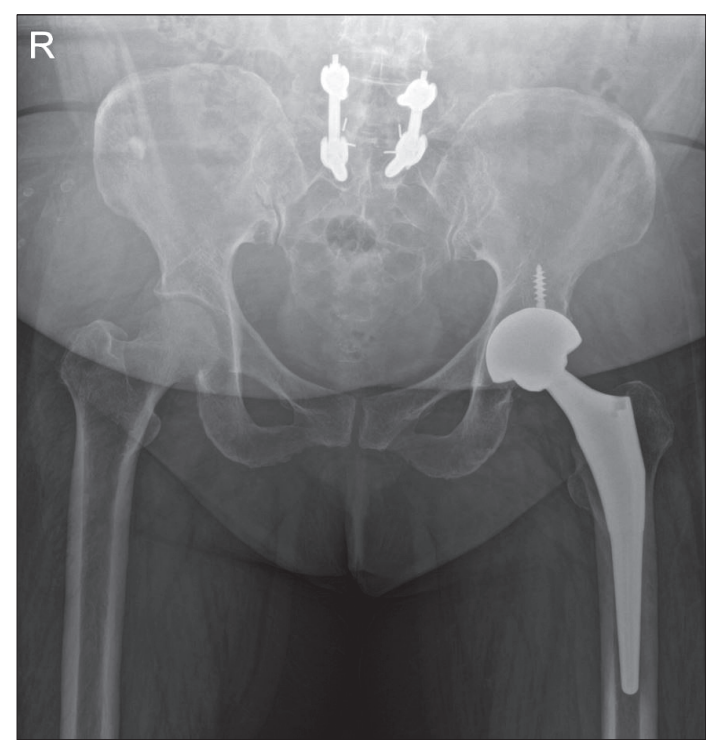

Fig. 2. Simple pelvis anteroposterial radiograph of patient with sciatic nerve palsy that occur after significant offset increase following total hip replacement. 
the ultrasound during the follow up. Change in the offset may also be a potential factor for developing the sciatic neruropathy [10]. Theoretically the sciatic nerve may stretch in proportion to the increased offset. In our series, we noticed the offset was increased in just one case. The case was 64 year old patient who had $2.4 \mathrm{~cm}$ lengthening of the joint due to joint instability (Fig. 2). We believe increased offset maybe a reason for the symptom in patient but the patient did not agree to undergo further procedure and the symptom remained in this patient. As the increase offset was noticed in only one case, we were unable to either confirm or deny that this is the risk factor for the poor prognosis. Furthermore, number of other reasons has been suggested such as tight piriformis repair or heterotopic ossification but we were unable to confirm this in our series as currently there is no tool to assess these factors [11-13].

We acknowledge that there are number of limitations to the current study. Firstly, the number of our cohort is too small. While our study is one of the largest series reported, the number is not sufficient to perform multivariate analysis. Therefore, the risk factor may change if larger series with multivariate analysis is performed. However, due to the scarcity of the sciatic nerve palsy following hip arthroplasty, we think such study design will be very difficult to proceed. Secondly, we were unable to confirm whether the sciatic nerve palsy has occurred due to direct or to indirect injury. It is likely that the result of the indirect injury will have better outcome than that of the direct injury where the nerve is actually penetrated or tethered by the retractor. However, none of the operation record described this and we were unable to confirm this.

In conclusion, this study indicates that not all sciatic nerve palsy may recover and thus it is important to warn the patient with this potential problem prior to the surgery. Also, we recommend proper rehabilitation should be developed for these patient populations.

Nevertheless, this study indicates that not all sciatic nerve palsy may recover and thus it is important to warn the patient with this potential problem prior to the surgery. Also, we recommend proper rehabilitation should be developed for these patient populations.

\section{ACKNOWLEDGEMENTS}

This study was supported by research fund from Chosun University Hospital, 2017.

\section{CONFLICT OF INTEREST}

No potential conflict of interest relevant to this article was reported.

\section{REFERENCES}

1. Brown GD, Swanson EA, Nercessian OA. Neurologic injuries after total hip arthroplasty. Am J Orthop (Belle Mead NJ) 2008;37:191-7.

2. Butt AJ, McCarthy T, Kelly IP, Glynn T, McCoy G. Sciatic nerve palsy secondary to postoperative haematoma in primary total hip replacement. J Bone Joint Surg Br 2005;87: 1465-7.

3. Simon JP, Van Delm I, Fabry G. Sciatic nerve palsy following hip surgery. Acta Orthop Belg 1993;59:156-62.

4. Farrell CM, Springer BD, Haidukewych GJ, Morrey BF. Motor nerve palsy following primary total hip arthroplasty. J Bone Joint Surg Am 2005;87:2619-25.

5. Park JH, Hozack B, Kim P, Norton R, Mandel S, Restrepo $\mathrm{C}$, et al. Common peroneal nerve palsy following total hip arthroplasty: prognostic factors for recovery. J Bone Joint Surg Am 2013;95:e55.

6. Zappe B, Glauser PM, Majewski M, Stöckli HR, Ochsner PE. Long-term prognosis of nerve palsy after total hip arthroplasty: results of two-year-follow-ups and long-term results after a mean time of 8 years. Arch Orthop Trauma Surg 2014; 134:1477-82.

7. de Bruijn IL, Geertzen JH, Dijkstra PU. Functional outcome after peroneal nerve injury. Int J Rehabil Res 2007;30:333-7.

8. Warner MA, Warner ME, Martin JT. Ulnar neuropathy. Incidence, outcome, and risk factors in sedated or anesthetized patients. Anesthesiology 1994;81:1332-40.

9. Edwards BN, Tullos HS, Noble PC. Contributory factors and etiology of sciatic nerve palsy in total hip arthroplasty. Clin Orthop Relat Res 1987;(218):136-41.

10. Eggli S, Hankemayer S, Müller ME. Nerve palsy after leg lengthening in total replacement arthroplasty for developmental dysplasia of the hip. J Bone Joint Surg Br 1999;81:8435.

11. Uchio Y, Nishikawa U, Ochi M, Shu N, Takata K. Bilateral piriformis syndrome after total hip arthroplasty. Arch Orthop Trauma Surg 1998;117:177-9. 
12. Kanda A, Kaneko K, Obayashi O, Mogami A, Morohashi I. Treatment of postoperative sciatic nerve palsy after total hip arthroplasty for postoperative acetabular fracture: A case report. Ann Med Surg (Lond) 2016;11:39-41.
13. Lohana P, Woodnutt DJ, Boyce DE. Sciatic nerve palsy-a complication of posterior approach using enhanced soft tissue repair for total hip arthroplasty. J Plast Reconstr Aesthet Surg 2010;63:e400-1. 